\title{
The constancy of object orientation: Compensation for ocular rotation*
}

\author{
SHELDON M. EBENHOLTZ $\dagger$ and KENNETH R. PAAP \\ Univerdity of Wisconsin, Medison, Wisconsin 53706
}

\begin{abstract}
When a thin horizontal line is displaced, either left or right of straight ahead, or when a vertical line is displaced up or down; systematic changes occur in the binocular disparity associated with the target. In three experiments, Ss matched the orientation of displaced targets with a variable comparison line. Estimates of apparent displacement with a pointing technique were also made. Since head position was fixed, apparent displacement was mediated by the angle of ocular rotation. Near perfect matches were made with vertical targets, but horizontal targets produced errors suggestive of underestimation of apparent displacement. However, the pointing data did not yield clear evidence for this view. Control data denied the possible role of the induced effect (IE) in matching the horizontal targets, and the results were discussed in the context of orientation constancy based upon compensation for displacement.
\end{abstract}

When a horizontal line target in the frontal plane is displaced horizontally or when a vertical target is displaced vertically fromithe straight-ahead position, the respective proximal patterns, assuming a fixed head position, will be systematically transformed. The displaced target will produce a pattern of stimulation equivalent to that of a nondisplaced target, seen with the eyes in primary position, i.e., straight ahead, but rotated about a horizontal or vertical axis, depending upon whether the direction of displacement was vertical or horizontal, respectively. The equivalence between displaced and rotated targets, respectively, holds not only for binocular disparity, but extends to monocular aspects of stimulation, such as perspective foreshortening, as well. The retinal patterns associated with a laterally displaced, horizontal target, together with a frontally viewed, rotated target producing the equivalent retinal projection, are represented in Fig. 1. By comparing the projections of the displaced target on the left with those of the rotated stimulus on the right, the equivalence of the two binocular representations can be demonstrated. Figure 1 also shows that equivalent monocular aspects of stimulation, such as retinal image size, are produced by, e.g., the right-eye projections of the displaced and rotated targets, respectively. It would appear that unless information was available to $S$ concerning the magnitude of ocular rotation; and hence the extent of target displacement, gross errors would occur in the perception of the spatial orientation of objects.

The geometrical basis for the claim of equivalent binocular disparities is further developed in Fig. 2 for the case of a laterally displaced horizontal line target seen in asymmetrical convergence. Analysis of vertical

-This research was supported by Grant MH 13006 from the National Institute of Mental Health. The authors thank Lynn Shebilske and Janine Pyrek for their aid as project assistants and Wilinm Epstein for making available the afocal magnifying lenses used in Experiment III.

tThe manuscript was prepared during the first author's leave as a Killam Senior Fellow at Dalhousic University. displacement follows analogous considerations and, except for the problem of cyclofusion, will not be discussed separately. The point of fixation is at the center of the line target, and Vieth-Muller (V-M) circles have been drawn through the fixation points and the nodal points of the two eyes. Two planes have been drawn tangent to the V-M circles, one at each point of fixation, to represent the reference surfaces against which target rotations are determined. With the eyes straight ahead, points on the tangent plane fall increasingly behind the $\mathbf{V}-\mathbf{M}$ circle, producing a pattern of increasing disparities to the right and left of the fixation point. This distribution of disparities corresponds to a frontal plane when the eyes are symmetrically converged. However, with the asymmetrical convergence, although the tangent surface again yields a symmetrical distribution of disparities to the right and left of the fixation point, the reference surface no longer is coincident with the frontal plane. With asymmetrical convergence to the left, a clockwise rotation relative to the tangent plane is required to place a target stimulus in the frontal plane, and for rightward displacements, counterclockwise rotations are necessary. Figure 2 illustrates the further point that a comparison stimulus seen straight ahead would have to be rotated clockwise from its reference plane in order to match the distribution of disparities produced by a horizontal target displaced to the left. ${ }^{1}$ Thus, if disparity matching was the primary basis for matching the orientations of the target and comparison stimuli, respectively, constancy and veridical orientation perception would not prevail. On the other hand, the occurrence of orientation constancy would imply that a given input, e.g., a pattern of binocular disparities, is evaluated in terms of the degree of ocular rotation required to fixate the target. ${ }^{2}$

The magnitude of rotation of the comparison stimulus from the tangent plane represents the degree of compensation entailed by the present type of ofientation constancy. For a target displaced $25 \mathrm{deg}$ left, 
$c$

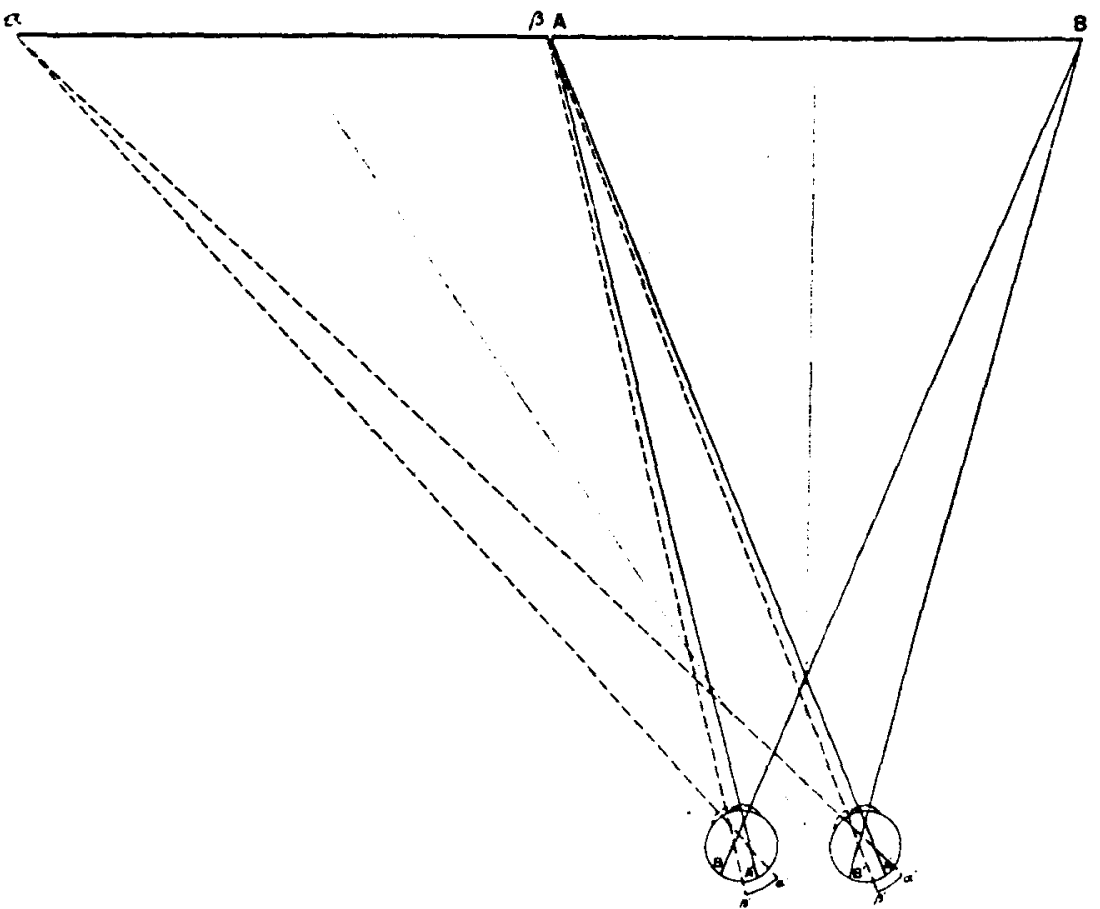

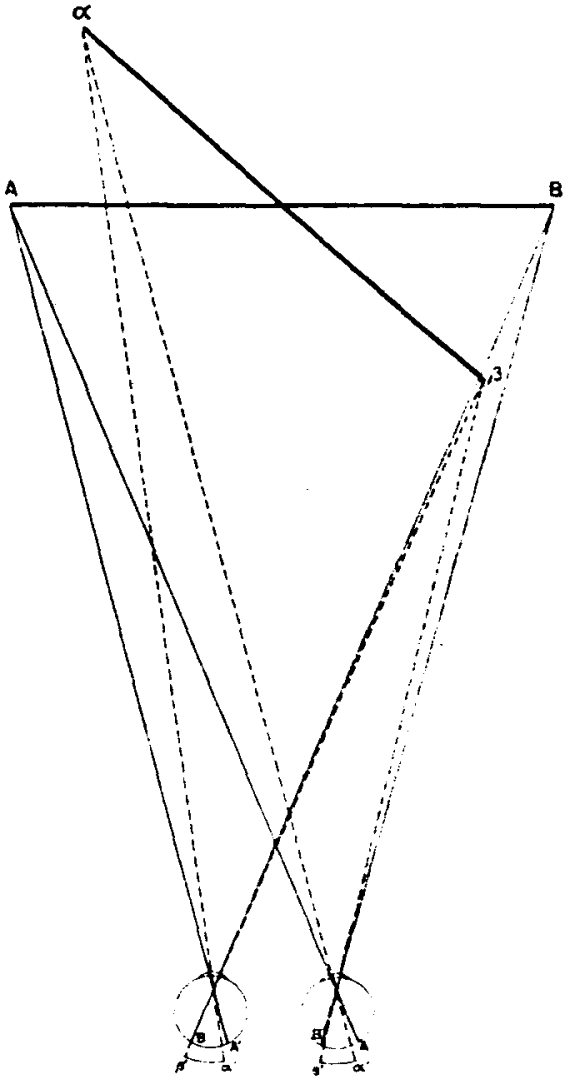

Fig. 1. The binocular equivalence of displaced and rotated targets. The retinal projections of Line $A B$ are the same size in each eye. However, the projections of the displaced target, $\alpha \beta$, on the left produce a larger image in the left eye than in the right eye. On the right, the left- and right-eye projections of the rotated target, $\alpha \beta$, produce the same differences as the displaced target on the left.

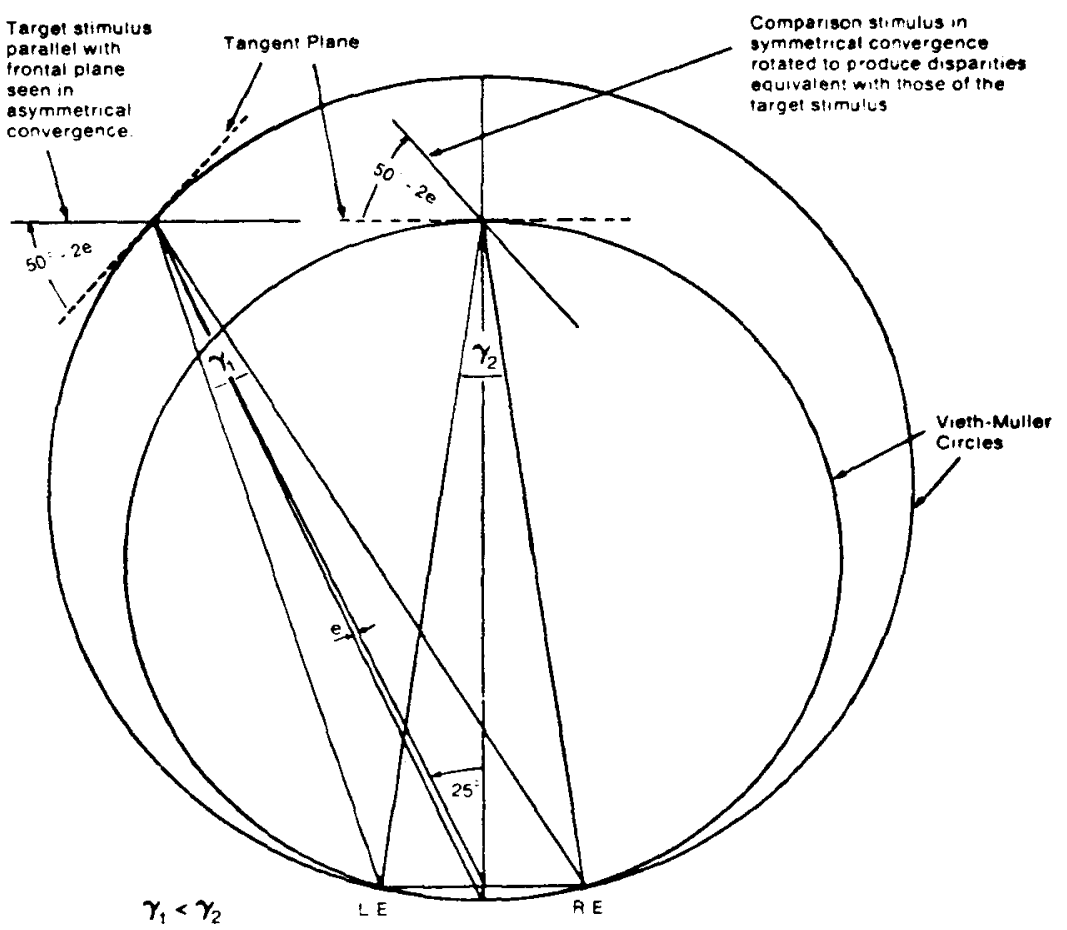

Fig. 2. A displaced frontal plane target and a comparison stimulus adjusted to produce the equivalent binocular disparity. The tangent planes are assumed to represent reference surfaces for depth perception. 
as represented in Fig. 2, the reference plane will be rotated from a frontal-plane orientation by somewhat less than two times that quantity. This results from the procedure of measuring the displacement from the midpoint of the interocular axis. Since the apex of the displacement angle does not lie on either V.M circle, the arc intersected by the two arms of the displacement angle is less than $50 \mathrm{deg}$ by the small quantity $2 \mathrm{e}$. The calculation of the magnitude of compensation thus depends on the orientation of the reference plane. If, e.g., the plane perpendicular to the bisector of the interocular axis is taken as the reference surface (Ogle, 1964 , p. 202), then the degree of compensation would be exactly half of that based upon the tangent plane, i.e., $25 \mathrm{deg}-\mathrm{e}$. This is a convenient result, for it approximates closely the displacement angle and suggests the simple rule that the magnitude of compensation is equal to the degree of ocular rotation from the primary position. Unfortunately, the question of the choice of reference surfaces remains unresolved, and consequently the precise degree of compensation in orientation constancy cannot be assessed from geometrical considerations alone.

There is yet an additional problem to be considered in specifying the degree of compensation, namely the question of the invariance in the shape of the empirical horopter with changes in the direction of gaze. The V-M circle represents a geometric abstraction of the distribution of points in space, the retinal images of which are presumed to stimulate common visual directions in each eye. Accordingly, the V-M circle is commonly regarded as the locus of points productive of zero disparity, i.e., the image of any point on the V-M circle will stimulate corresponding points on each retina. There is, in contrast, the empirically resolvable question of the locus in space of points whose retinal images actually do stimulate common visual directions. The nonius horopter, when empirically determined with the eyes fixated in symmetric convergence, could, in principle, have a shape other than circular and, although this possibility is not of particular consequence in the present context, ${ }^{3}$ it is of significance to consider that the shape could change as the eyes asymmetrically converge such that frontal plane targets would continue to stimulate corresponding retinal points. There would thus be no need for any compensation process. Although this is an extreme possibility, it should be noted that any flattening of the horopter in asymmetrical convergence would diminish the magnitude of compensation necessary for orientation constancy. Ogle (1964) summarized research on this question by noting that points on the empirical horopter, based upon the criterion of common visual directions, i.e., the nonius horopter, with the eyes in asymmetrical convergence would seem to ". . . lie close to the Veith-Muller circle at the fixation point [p. 214]." Thus, there is evidence that the V-M circle offers an excellent approximation to the nonius horopter and, perhaps more importantly, that

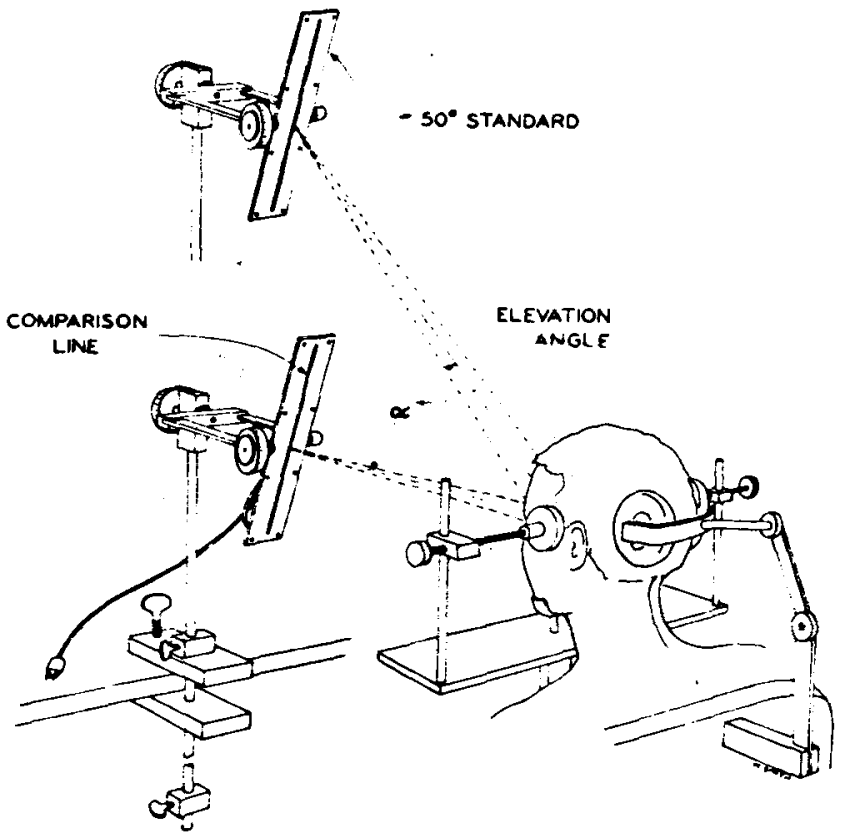

Fig. 3. A vertically displaced standard with the comparison at eye level. The $-50-\mathrm{deg}$ orientation was used only in the screening task.

the shape of the empirical horopter does not change in asymmetric convergence. Recent studies (Shipley \& Rawlings, 1971) also support this latter conclusion, but indicate further that the locus of common visual directions is considerably more complicated than the simple V-M curve. It may be concluded that, because of the unresolved state of the problem of the appropriate reference plane for stereoscopic depth and because of the lack of complete identity between the empirical nonius horopter and the V-M circle, especially at distances less than $1 \mathrm{~m}$, the exact degree of compensation cannot now be specified in some a priori manner. However, casual observation, as well as horopter studies of the apparent frontoparallel plane and the apparent normal plane in asymmetrical convergence (e.g., Shipley \& Rawlings, 1971; Ogle, 1964), suggests that some form of compensation associated with the asymmetrical direction of gaze does indeed take place, i.e., S's settings depart from both the V-M circle and the empirical nonius horopter in a veridical direction. Thus, Ogle (1964) noted that ". . . the subjective criterion of the apparent normal plane utilizes an entirely different set of retinal elements in the two eyes in asymmetrical convergence from that utilized for symmetrical convergence. Furthermore, those used in asymmetrical convergence are not corresponding retinal elements [p. 215]."

Because the problem of the apparent orientation of displaced stimuli represents one instance of a large class of spatial constancy phenomena and one aspect of the problem of the constancy of object orientation (Ebenholtz, 1970; Day \& Wade, 1969), it extends beyond the domain of horopter determinations. 


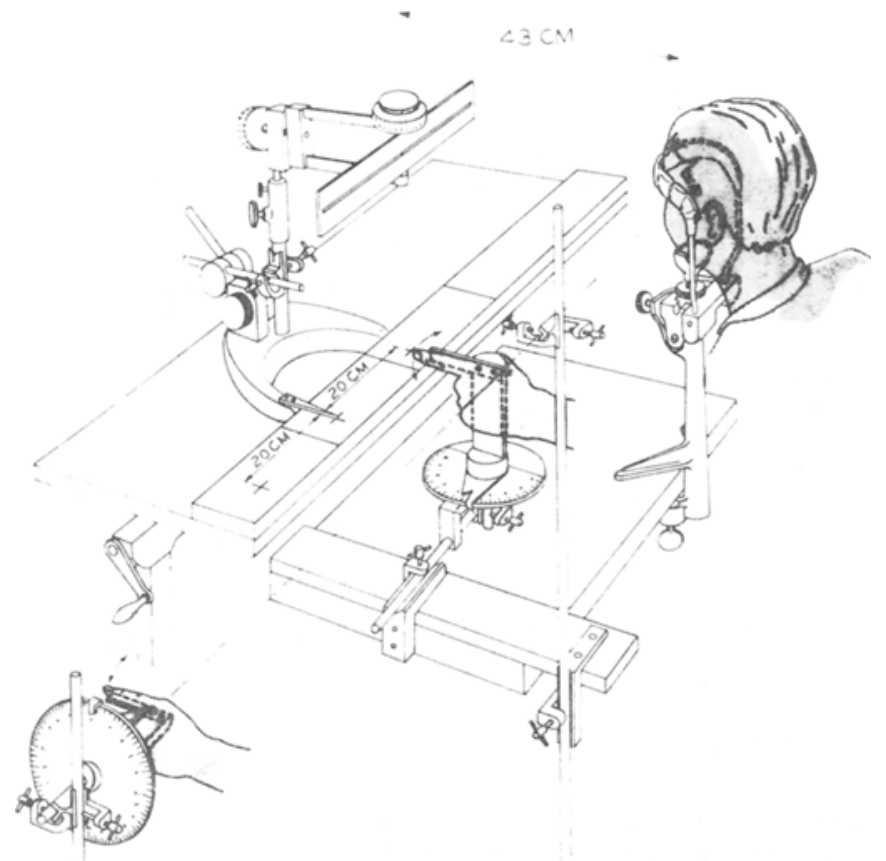

The plane within which displacement was measured, i.e., vertical and horizontal, was varied between $\mathrm{Ss}$, as was the orientation (e.g., left side away vs frontoparallel) of the standard stimulus.

In Experiment 1, Ss were tested for stereoacuity on a Bausch and Lomb orthorater. All but 2 Ss satisfied the criterion of at least one correct response, and these were replaced for a final sample size of 16 . In all three experiments, the screening task required $S$ to match a standard line target with a successively presented comparison, both of which were shown at eye level and straight ahead of $S$. The rotation of the screening targets was in the same plane as the subsequently presented test targets, i.e., either in the median or horizontal planes. In the former case, the standard line used during the screening task was oriented at $-25 \mathrm{deg}$, top toward $\mathrm{S}$, and in the latter case, it was at $-25 \mathrm{deg}$, left side toward S. None of these orientations was again used in the experiment proper. This screening procedure served the function of reducing variability by introducing $S$ to a task similar to that required in the main part of the experiment and by permitting some $S$ selection to occur. Ss made two matches of the standard, one each from stating positions $15 \mathrm{deg}$ on either side of the correct orientation. An average error over $6 \mathrm{deg}$, or a range in excess of $10 \mathrm{deg}$, was used to disqualify 8 Ss in Experiment II, ${ }^{5}$ for a usable total of 36 , and $5 \mathrm{Ss}$ in Experiment III, in which 24 Ss took part.

After completing the experimental matching tasks, Ss of Experiments II and III were presented with the standard stimuli at the same displacement angles, as in the matching task, but were required to indicate the magnitude of the apparent target displacement by pointing with the left index finger while fixating the spot at the center of the luminous line. Figure 4 illustrates the pointing apparatus and its location relative to $S$. The apparatus, essentially, is a half-cylinder with a flat plate to serve as a guide for the pointing finger. The cylinder rotated about its longitudinal axis, which, in the case of the vertically displaced targets, was placed to the left of $S$ at eye level in order to correspond, approximately, with the (horizontal) axis of vertical eye movements. This is shown to the left in Fig. 4. Note should be taken of the fact that the apparatus constrained $S$ from pointing directly at the targets, since they were always in the median plane whereas the index finger rotated in a parallel plane approximately $6 \mathrm{~cm}$ to the left of the median plane. In this position, however, $S$ could rotate his wrist in order to match the angle of ocular elevation or depression produced by fixating the center point of the target. Two settings of the pointer were made by $S$ for each displacement position of the line, with the starting position of the pointer at $20.5 \mathrm{deg}$ on either side of the correct direction. The instructions explicitly directed $S$ to point his index finger at that position in space the vertical locus of which would correspond with the fixation target. Thus, for targets straight ahead, the correct pointer setting would yield an index finger parallel with the floor. For displacements $25 \mathrm{deg}$ above and below straight ahead, the pointer had to be rotated $25 \mathrm{deg}$ in the proper direction.

For measuring the apparent displacement of horizontal targets, the pointing device was oriented to rotate around a vertical axis placed about $10 \mathrm{~cm}$ to the left of the median plane and $23 \mathrm{~cm}$ in front of $\mathrm{S}$. The index finger thus rotated in a horizontal plane about $20 \mathrm{~cm}$ below eye level and hence below the level of the line stimuli. This is represented in the center of Fig. 4. Since Ss could not point directly at the center of the targets, they were instructed to rotate the wrist (left or right) so that the index finger was felt to be pointing at a position in space right below the point being fixated. As in the condition of vertical target, displacement starting positions for the pointer settings were at $20.5 \mathrm{deg}$ on either side of the correct position. Because of the forward placement of the device relative to the plane containing the (vertical) axes for horizontal ocular rotation, and also because of the lateral displacement of the pointer axis from S's median plane, correct pointer settings, unlike the vertical displacement conditions, did not correspond to the actual magnitude of ocular rotation. Thus, for a target 
Table 1

Mean Errors, Mean Absolute Scores, and Standard Errors ( $\sigma \mathrm{m})$ for Two Orientations of the Standard (0 Deg and +50 Deg) and Two Elevations (O Deg and $22.7 \mathrm{Deg}$ ) of the Comparison (Target Rotation and Displacement was in the Median Plane)

\begin{tabular}{|c|c|c|c|c|c|c|c|}
\hline & & \multicolumn{6}{|c|}{ Orientation of Standard Line (Presented at Eye Level) } \\
\hline & & $\begin{array}{l}\text { Mean } \\
\text { Error } \\
\text { Score }\end{array}$ & $\begin{array}{c}\text { Mean } \\
\text { Absolute } \\
\text { Score }\end{array}$ & $\sigma_{\mathrm{m}}$ & $\begin{array}{l}\text { Mean } \\
\text { Error } \\
\text { Score }\end{array}$ & $\begin{array}{c}\text { Mean } \\
\text { Absolute } \\
\text { Score }\end{array}$ & $\sigma_{\mathrm{m}}$ \\
\hline $\begin{array}{l}\text { Displacement } \\
\text { of Compari- } \\
\text { son Line }\end{array}$ & $\begin{array}{l}\text { Above }(22.7 \mathrm{Deg}) \\
\text { Eye Level }(0 \mathrm{Deg})\end{array}$ & $\begin{array}{r}0.0 \\
-1.2\end{array}$ & $\begin{array}{r}0.0 \\
-1.2\end{array}$ & $\begin{array}{l}1.4 \\
.96\end{array}$ & $\begin{array}{r}1.6 \\
-1.8 \\
\end{array}$ & $\begin{array}{l}51.6 \\
48.2\end{array}$ & $\begin{array}{l}1.5 \\
1.1\end{array}$ \\
\hline
\end{tabular}

Note: - indicates top toward $S$, indicates top away from $S$

whose center was directly in front of $S$, i.e., 0 deg, the pointer had to be rotated to the right, from a straight-ahead position, by $23.5 \mathrm{deg}$. For a target with a midpoint $25 \mathrm{deg}$ to $S$ 's left, the pointer had to rotate left $23.5 \mathrm{deg}$, and for targets displaced rightward $25 \mathrm{deg}$, a rightward pointer rotation of $52.5 \mathrm{deg}$ was required.

At no time during the experiment was the pointer, $S$ 's finger, or anything but the line target visible to $S$. Markings on the pointer and the comparison stimuli were in dgrees of arc estimated to $.5 \mathrm{deg}$. Ss began the experiment after about $30 \mathrm{sec}$ in the dark.

\section{EXPERIMENT I}

\section{Design}

In Experiment I, the line stimuli rotated in the median plane with the standard always shown at eye level $(0 \mathrm{deg})$ and the comparison either at eye level at a distance of $61 \mathrm{~cm}$ from $\mathrm{S}$ or elevated $22.7 \mathrm{deg}$. There were two groups of eight Ss each, one of which was shown a vertical standard (Group V), the remaining Ss being given a standard with an orientation at $+50 \mathrm{deg}$, top tilted away from $S$ (Group T). The standard was presented for a $30-\sec$ inspection period, followed by a $90-\mathrm{sec}$ interval with eyes covered, during which $E$ rotated the line to the appropriate starting position and placed the comparison at the required elevation. The time used by $S$ to instruct $E$ to adjust the comparison was unlimited, but rarely exceeded $30 \mathrm{sec}$. An additional $30 \mathrm{sec}$ was required to adjust the stimulus for the next presentation of the standard. In all, each $S$ engaged in four matching trials, i.e., two at each elevation of the comparison, alternating between eye level and $22.7 \mathrm{deg}$ above. Half the Ss started their match with the elevated comparison, the remaining Ss starting with the comparison at eye level. The order of starting positions (top toward $S$ or top away) was also counterbalanced over Ss.

The instructions emphasized the apparent rather than objective orientation, and $S$ was encouraged to note the orientation of the line egocentrically, i.e., relative to the sagittal axis of his head.

\section{Results}

\section{Screening Task}

The two groups were compared in terms of their performance on the screening task. Mean errors of -1.1 and -2.6 were produced by Groups $\mathrm{V}$ and $\mathrm{T}$, respectively. Thus, Ss' settings were tilted slightly beyond the correct $-25-\mathrm{deg}$ orientations. However, there were no significant differences between groups; hence, they may be considered to be well matched in terms of the ability to perform the orientation matching task.

\section{Matching Data}

For the main task data, an analysis of variance was performed with displacement of the comparison (eye level or above) as a within-S factor, orientation of the standard (vertical or $+50 \mathrm{deg}$ ) varying between Ss. Both the error scores, i.e., the deviation of the setting from a perfect match, and absolute scores, i.e., the actual orientation of the S's match relative to the direction of gravity, were analyzed. The mean scores and standard errors are represented in Table 1. Results of the analysis of absolute scores showed a significant effect of orientation, $F(1,14)=2,898.01, p<.01$. This indicates that Ss clearly discriminated in their settings between the vertical and 50-deg standards. Neither displacement, $F(1,14)=2.57, p>.05$, nor the interaction of displacement with orientation, $F(1,14)=0.54, p>.05$, was significant. Analysis of the error scores yielded identical variance terms for the within-S variables (i.e., displacement and interaction). Orientation did not yield significant effects, $F(1,14)=0.28, p>.05$, and $t$ tests failed to reveal that individual means differed significantly from zero. The results of Experiment I show that line targets oriented in S's median plane and displaced vertically yield nearly perfect constancy. This suggests that compensation for the distortions in the retinal projections that normally accompany ocular elevation was virtually complete.

\section{EXPERIMENT II}

Experiment II extended the conditions of the first experiment by examining the effects of displacement in the median plane both above and below eye level. Displacement of horizontal targets to the left and right of straight ahead also was explored. The apparatus was the same as that previously described. However, the distance of the target from $S$, measured in Ss' median plane at eye level, was reduced to $43 \mathrm{~cm}$.

\section{Design}

Thirty-six undergraduates who had not previously taken part 
Table 2

Mean Errors, Mean Absolute Scores, and Standard Errors $\left(\sigma_{\mathrm{m}}\right)$ for Two Orientations of the Standard $(0 \mathrm{Deg}$ and $+40 \mathrm{Deg})$ and Three Displacement Angles (Eye Level, 25 Deg Above, 25 Deg Below). Target Rotation and Displacement was in the Median Plane.

\begin{tabular}{|c|c|c|c|c|c|c|c|}
\hline & & \multicolumn{6}{|c|}{ Orientation of Standard Line (Presented at Eye Level) } \\
\hline & & \multicolumn{3}{|c|}{0 Deg (Vertical) } & \multicolumn{3}{|c|}{ +40 Deg (Top Away) } \\
\hline & & $\begin{array}{l}\text { Mean } \\
\text { Error } \\
\text { Score } \\
\end{array}$ & $\begin{array}{c}\text { Mean } \\
\text { Absolute } \\
\text { Score }\end{array}$ & $\sigma_{\mathbf{m}}$ & $\begin{array}{l}\text { Mean } \\
\text { Error } \\
\text { Score }\end{array}$ & $\begin{array}{c}\text { Mean } \\
\text { Absolute } \\
\text { Score } \\
\end{array}$ & $\sigma \mathrm{m}$ \\
\hline $\begin{array}{l}\text { Displacement } \\
\text { of Standard } \\
\text { Line }\end{array}$ & $\begin{array}{l}\text { Above }(25 \mathrm{Deg}) \\
\text { Eye Level }(0 \mathrm{Deg}) \\
\text { Below }(-25 \mathrm{Deg})\end{array}$ & $\begin{array}{l}0.2 \\
1.1 \\
3.3\end{array}$ & $\begin{array}{l}0.2 \\
1.1 \\
3.3\end{array}$ & $\begin{array}{l}1.6 \\
1.6 \\
1.2 \\
\end{array}$ & $\begin{array}{r}0.8 \\
-0.1 \\
0.1 \\
\end{array}$ & $\begin{array}{l}40.8 \\
39.9 \\
40.1 \\
\end{array}$ & $\begin{array}{l}2.0 \\
1.1 \\
1.9 \\
\end{array}$ \\
\hline
\end{tabular}

Note: - indicates top toward $S,+$ indicates top away from $S$; comparison line at eye level

in orientation studies were distributed into four groups of nine Ss each. For two groups, displacement of the standard was in a horizontal plane (Condition $\mathrm{H}$ ) at eye level, with the center point of the line straight ahead of $S$ or $25 \mathrm{deg}$ to the right and left. For these groups, the comparison line rotated in the horizontal plane around a vertical axis. The remaining two groups were shown standards that rotated in the median plane about a horizontal axis and with a center point at eye level or displaced vertically (Condition V) $25 \mathrm{deg}$ above or below eye level. Within each displacement condition, there was a different orientation of the standard for each of the two groups. In Condition $\mathrm{H}$, one standard was oriented parallel with S's frontal plane, while the other was rotated from the frontal plane $40 \mathrm{deg}$, with the left side away from $S$. In Condition $V$, one group was exposed to a vertically oriented standard, i.e., in line with the direction of gravity. The remaining group was shown a standard with the top tilted away from S by 40 deg. In both Condition V and Condition $\mathrm{H}, \mathrm{S}$ matched the apparent orientation of the standard with the comparison line, the lat ter always being shown straight ahead of $S$ with the pivot point at eye level. The Ss were assigned to conditions alternately on the basis of arrival at the laboratory.

The experimental matching trials began immediately after the previously described screening task. Exposure time for the standard was $15 \mathrm{sec}$, with the matching trial following a $30-\mathrm{sec}$ period with the eyes covered. The setting required approximately $30 \mathrm{sec}$, and an additional $45 \mathrm{sec}$ was needed to arrange the standard for the next match. The order of testing was counterbalanced over Ss by assigning three Ss to each of the three sequences of the displacement angles (e.g., a, b, c; b, c, a; c, $\mathrm{a}, \mathrm{b})$. The $\mathrm{S}$ made one match with the standard at each of the three displacement positions and then repeated the sequence in reverse order. This yielded two matches for each displacement angle, viz, one each with the comparison at starting positions $15 \mathrm{deg}$ on either side of the correct setting.

All Ss were instructed to match on the basis of the apparent orientation of the target. For Condition V, S was instructed to judge the apparent tilt relative to the direction of gravity, as opposed to the egocentric frame of reference used in Experiment 1. In Condition $\mathrm{H}$, "apparent" instructions were again issued, but the frame of reference for tilt was the frontoparallel plane, described as a wall straight in front of $S$. The experiment began after $E$ answered all questions and $S$ was satisfied that he understood the task.

\section{Results}

The data of Conditions $\mathrm{V}$ and $\mathrm{H}$ were not directly compared in the following analyses because the conditions varied simultaneously in several ways. Thus, not only did Conditions $\mathrm{V}$ and $\mathrm{H}$ differ in the direction of displacement, but in the plane of rotation of the comparison stimuli and in the orientation of the targets as well.

\section{Screening Task}

In Condition $\mathrm{V}$, the group subsequently receiving the vertical standard (Group V) yielded a mean error of $0.8 \mathrm{deg}$. The group scheduled to receive the standard tilted at $+40 \mathrm{deg}$ (Group T) showed an error of $0.9 \mathrm{deg}$. The groups did not differ significantly from each other, nor was the average score significantly different from zero. In Condition $\mathrm{H}$, the group set to receive the horizontal standard (Group $\mathrm{H}$ ) showed a mean error of $-2.6 \mathrm{deg}$, left side rotated toward $\mathrm{S}$. The remaining group scheduled for the tilted standard (Group T) yielded a mean error of -0.1 . Again, there were no significant differences, nor was the combined mean significantly different from zero. It may be concluded that the two groups within each condition were well balanced in ability to perform the matching task.

\section{Matching Data, Vertical Displacement}

Analysis of variance was performed with orientation of the standard (upright and +40 deg top away) as the between-S factor and displacement (eye level, $25 \mathrm{deg}$ above and $25 \mathrm{deg}$ below) as the within-S variable. The data of both absolute and error scores are represented in Table 2. In the former case, orientation was highly significant, $F(1,16)=1,005.57, p<.01$, indicating that $S$ clearly differentiated between orientations of the standard. Neither displacement, $F(2,32)=0.39, p>.05$, nor the interaction of orientation and displacement was significant, $F(2,32)=0.68, p>.05$. The latter two terms were identical for the error score analysis, while orientation yielded $F(1,16)=1.09, p>.05$. Thus, there were no effects on apparent orientation attributable to shifts in the elevation or depression of gaze. These data are consistent with those of Experiment $I$ in showing nearly perfect constancy for targets displaced in the median plane.

\section{Matching Data, Horizontal Displacement}

Mean performance expressed both in absolute and 
Table 3

Mean Errors, Mean Absolute Scores, and Standard Errors $\left(\sigma_{\mathrm{m}}\right)$ for Two Orientations of the Standard (0 Deg and +40 Deg) with Displacement Angle as Indicated (Target Rotation and Displacement was in the Horizontal Plane at Eye Level)

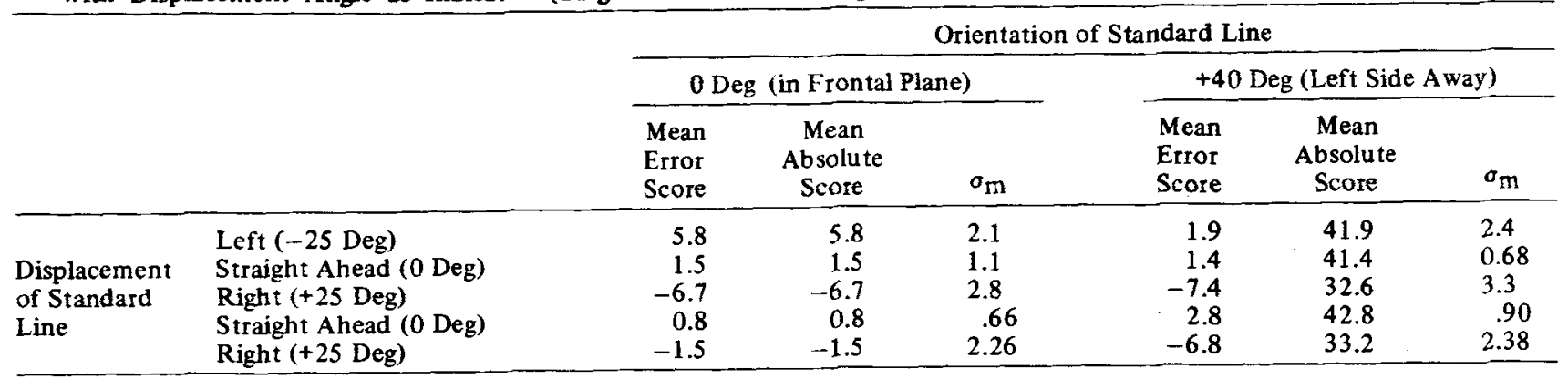

Note: - indicates left side toward $S$, indicates left side away from $S$; comparison line straight ahead of $S$

error scores is represented in the top three rows of Table 3 . The absolute scores show that orientation was again significant, $F(1,16)=895.54, p<.01$, indicating good discrimination between the targets. Displacement also proved to be significant, $\mathrm{F}(2,32)=10.53, \mathrm{p}<.01$, but the interaction was not, $F(2,32)=0.34, p>.05$. The outcome was, of course, identical for the error score analysis of displacement and the interaction term. However, the errors did not vary significantly with orientation, $\mathrm{F}(1,16)=1.47, \mathrm{p}>.05$.

The mean errors represented in Table 3 show an interesting pattern in that the errors were positive for targets displaced to the left and negative when targets were presented to $S$ 's right. This indicates that stimuli on the right appeared to be rotated right side away, while those on the left appeared to be rotated left side away. This outcome is consistent with an underestimation in the magnitude of the direction of ocular rotation. This appears to be true for both orientations of the target, although the effect is reduced for the tilted standard presented on the left.

\section{Pointing Data, Vertical Displacement}

In the pointing task, absolute scores were defined as deviations from a zero point. This point was achieved in Condition V, with S's finger parallel to the horizontal plane. Rotations of the wrist, and hence of the direction of the pointing finger above and below this plane, were scored as plus and minus, respectively. Error scores were defined as deviations from the correct direction, with negative scores indicating that $S$ had pointed below the correct position. The means and standard errors of both absolute and error scores are shown in Table 4. Analysis of variance of the absolute scores showed a significant effect of orientation, $F(1,16)=9.96$, and displacement, $F(2,32)=249.33, p<.01$ in both cases. The interaction term was not significant, $F(2,32)=0.32, p>05$. The significant displacement effect shows clearly that $S$ discriminated among the three displacement positions by pointing at differential spatial locations. Furthermore, the pointing directions were properly ordered from top to bottom relative to the correct positions, although, as Table 4 shows, there appears to be a large constant error operating, which caused $S$ to point considerably below the target. This tendency was greater for the vertical target than for the tilted one, and led to the significant effect of orientation. There is no ready explanation for this outcome. The error scores, i.e., deviations from the correct direction, also showed a significant orientation

Table 4

Pointing Data: Mean Errors, Absolute Scores and Standard Errors $\left(\sigma_{\mathrm{m}}\right)$ for Displaced and Rotated Targets

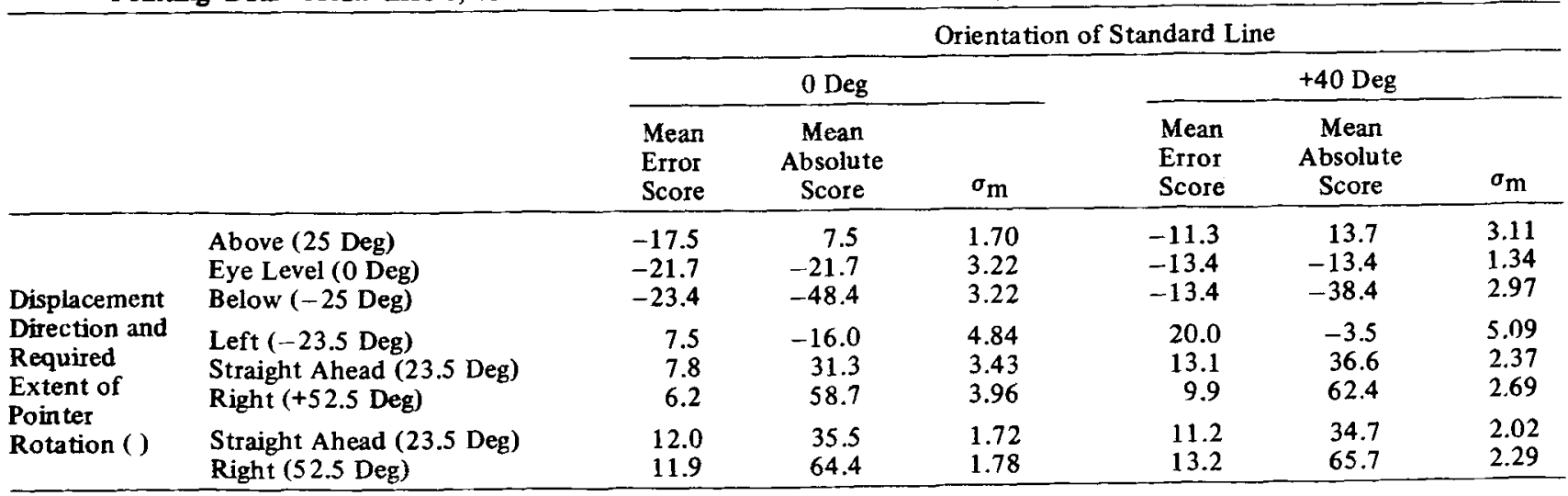

Note: - indicates below or left 
Table 5

Calculated and Obtained Mean Relative Angular Shift in Pointing Direction (Degrees) from Central to Peripheral Targets

\begin{tabular}{|c|c|c|c|c|c|c|c|c|}
\hline & \multicolumn{4}{|c|}{ Vertical } & \multicolumn{4}{|c|}{ Horizontal } \\
\hline $\begin{array}{l}\text { Calculated } \\
\text { Obtained-Experiment II } \\
\text { Obtained-Experiment III }\end{array}$ & $\begin{array}{l}25.0 \\
29.2\end{array}$ & $\begin{array}{l}-25.0 \\
-26.7\end{array}$ & $\begin{array}{l}25.0 \\
27.1\end{array}$ & $\begin{array}{l}-25.0 \\
-25.0\end{array}$ & $\begin{array}{l}-47.0 \\
-47.3\end{array}$ & $\begin{array}{l}29.0 \\
27.4 \\
28.9\end{array}$ & $\begin{array}{l}-47.0 \\
-40.1\end{array}$ & $\begin{array}{l}29.0 \\
25.8 \\
31.0\end{array}$ \\
\hline
\end{tabular}

Note: - indicates below or left

effect, $F(1,16)=9.96, p<.01$, however, neither displacement, $F(2,32)=1.52$, nor the interaction effect, $F(2,32)=0.32$, was significant, with $p>.05$ in each case. Thus, Ss were more accurate in pointing at the center of the tilted target than at the midpoint of the vertical one. Target displacement had no effect on the size of the pointing error.

\section{Pointing Data, Horizontal Displacement}

In Condition $\mathrm{H}$, absolute scores were determined with reference to a zero point represented by a finger oriented parallel with the median plane. Positions to the right and left were given plus and minus signs, respectively. Means and standard errors are represented in the middle three rows of Table 4. Analysis of variance of the absolute scores showed a significant effect of orientation, $\mathrm{F}(1,16)=6.33, \mathrm{p}<.05$, and displacement, $F(2,32)=153.66, p<.01$. The interaction was not significant, $F(2,32)=0.67, p>.05$. Analysis of error scores showed the identical $F$ ratios for orientation and the interaction of orientation with displacement as that based upon absolute scores. ${ }^{6}$ The error scores did not vary significantly with displacement. These results of both analyses indicate that, as in Condition V, Ss are capable of making a differential pointing response to the three displacement positions. Furthermore, there was evidence, again, of a constant error, this time to the right of the target. In addition, Ss were more accurate in pointing at the horizontal target, and this is reflected in the significant effect of target orientation.

The data of the matching task with horizontal displacement produced errors consistent with the hypothesis of underestimation in the magnitude of ocular rotation, i.e., in the apparent displacement of the target. Such an underestimation in ocular position has been reported by Hill (1972). However, because of the presence of a constant error, the pointing data do not provide direct evidence on this question. A further analysis, therefore, was attempted. To this end, the angular shift in the pointing finger, between the center and peripheral target positions, was calculated. Thus, the position of the finger when the center target was fixated was noted along with the position assumed by $S$ when pointing at each of the displaced targets. The angular difference between a displaced and a center-target finger direction in comparison with the actual or calculated angular finger displacement needed to point correctly was used to provide a measure of over- or underestimation. These data are represented in Table 5 for both the vertical and horizontal target-displacement conditions. In Condition $\mathrm{H}$, there would appear to be a slight tendency toward underestimation; however, the differences between the calculated and obtained shifts were not reliable. The most interesting outcome of this analysis is the remarkably close correspondence between the actual and obtained values, suggesting that, despite the constant error, the pointing response is a reliable indicator of relative angular displacement between targets.

Although the mean relative angular shift in pointing did not show significant underestimation, there remained the possibility that Ss might show a correlation between errors in the matching task ${ }^{7}$ and the degree of over- or underestimation in the pointing task. Rank order correlations were calculated for both vertical and horizontal conditions. These tended to be positive, but low and nonsignificant.

\section{EXPERIMENT III}

The results of the two experiments agree in showing a relatively high degree of orientation constancy, with near perfect compensation for vertically displaced targets and somewhat lower, but nevertheless substantial, compensation for horizontal displacements. This outcome is not at all consistent with a disparity matching model of the perception of object orientation. Analogous results occur in the settings of Ss attempting to place the horopter rods according to the criterion of the apparent normal plane (ANP) ${ }^{8}$ Such settings, made under conditions of asymmetrical convergence generally fall quite close to the true normal plane, but in so doing, the horopter rods yield a pattern of disparity different from that produced by ANP settings with the eyes symmetrically converged. Ogle (1964) attempted to account for these results with the induced effect (IE), viz, "When one increases the magnification of the image of the right eye in the vertical meridian by a suitable lens, an apparent distortion of space occurs in the sense that objects at the right of the fixation point appear nearer, those on the left farther-a clockwise rotation of 
the field [p. 223]." In viewing a target, e.g., asymmetrically to the right, it is true that the right-eye image would be larger than the left-eye image. Consequently, if this relative image magnification were only in the vertical dimension, the IE might be expected to occur. However, in fact, the image is magnified in the horizontal dimension as well as in the vertical, and horizontal image magnification in the right eye is known to produce an apparent rotation of the frontal plane, the part on the side of the right eye rotating away from $S$, viz, a counterclockwise rotation generally known as the geometric effect (GE) (Gillam, 1968; Ogle, 1964). It follows from these considerations that near veridical performance with the horizontal targets of Experiment II, or with the settings of the horopter apparatus to the ANP, can be deduced from the assumption that the IE and GE are simultaneously active and opposed effects of equal magnitude. Experiment III examined this cancellation hypothesis by directly testing for an induced effect with the horizontally oriented targets.

\section{Design}

After the screening task with the -25-deg standard (right side away), the two groups of $12 \mathrm{Ss}$ each were exposed to the horizontal targets used in Experiment II. Group F matched a target oriented at 0 deg, i.e., parallel with the frontal plane, whereas Group $T$ received the standard tilted at $+40-\mathrm{deg}$ orientation (left side away). Only two displacement angles were used, i.e., the standard was shown straight ahead and 25 deg to the right. As in the previous experiment, the comparison was always shown straight ahead of $S$. The remaining procedure and exposure times were also the same as in Experiment II; however, the interval between the end of the exposure period and the presentation of the comparison was reduced from 30 to $15 \mathrm{sec}$. After the matching task, the pointing apparatus again was used to obtain estimates of apparent displacement and the procedure was exactly that described in Experiment II.

The test for the IE followed the pointing task. An overall size lens (OSL), producing a magnification of $4 \%$ in all meridional directions, $\theta$ was worn over $S$ 's right eye while $S$ viewed the standard (either at 0 or $+40 \mathrm{deg}$ ) with no displacement, i.e., located straight ahead. Viewing the standard through the OSL alternated with direct viewing, i.e., with no lens (NL), for a total of four matches. The comparison target was always viewed directly, without any lens. Immediately after these trials were completed, two additional matches were made, this time with a meridional size lens at axis $180 \mathrm{deg}$ placed over the right eye. This lens, referred to as the inducing lens (IL), magnified the image in the vertical meridian by $4 \%$, and hence represented conditions suitable for the IE. If the IE were to occur, the IL should cause $S$ to perceive the standard as if a $4 \%$ horizontal magnifier (axis $90 \mathrm{deg}$ ) were worn over the left eye, viz, the targets should appear rotated left side away from their correct orientations. Viewing through the OSL should cause the GE and IE to cancel and hence performance here should not differ when compared with the NL condition.

\section{The Geometric Effect}

Ogle (1964) developed a formula for computing the apparent angular rotation, $\theta$, of the frontal plane in terms of the ratio of the sizes of the magnified and normal images, $M$, the viewing distance, $y$, and one-half the interpupillary axis, a. This formula is given as $\tan \theta=$ $[(M-1) / 2 M][y / a]$. In the present experiment, where $M$ $=1.04, y=43 \mathrm{~cm}$, and a is taken as $3.3 \mathrm{~cm}, \tan \theta=$ .2506 . Hence $\theta \cong 14 \mathrm{deg}$. Accordingly, in the absence of an IE, the OSL ${ }^{10}$ should cause the horizontal standard to appear rotated right side away, $14 \mathrm{deg}$. On the other hand, assuming an IE, the magnitude should also be $14 \mathrm{deg}$, but in the opposite direction. These effects may be expected for standards viewed in the frontal plane, but Ogle's formula is not directly applicable for targets at other orientations. This can be shown by noting that the GE is directly proportional to the change in binocular disparity $(\Delta \eta)$ produced by the introduction of the aniseikonic lens. Consider the initial disparity $\left(\eta_{0}\right)$ as equal to the difference in the horizontal dimensions of the left $\left(\alpha_{1}\right)$ and right $\left(\alpha_{2}\right)$ eye images, respectively, of the target. With the lens over the right eye, the disparity changes to $\eta_{1}$ because of the change in $\alpha_{2}$. The difference between $\eta_{0}$ and $\eta_{1}$, i.e., $\Delta \eta$, is responsible for the GE.

Thus,

$$
\begin{aligned}
\Delta \eta=\eta_{0}-\eta_{1} & =\left(\alpha_{1}-\alpha_{2}\right)-\left(\alpha_{1}-\mathrm{M} \alpha_{2}\right) \\
& =\mathrm{M} \alpha_{2}-\alpha_{2} \\
\Delta \eta & =\alpha_{2}(\mathrm{M}-1)
\end{aligned}
$$

Since any rotation out of the frontal plane will decrease $\alpha_{2}$, it follows that $\Delta \eta$, and hence the GE, will be at a maximum for frontal targets and will be reduced by targets at other orientations. In order to infer the GE for the target oriented at $+40 \mathrm{deg}$ (left side away), it was first necessary to solve Ogle's formula for $M$. This yielded

$$
\mathrm{M}=\frac{2 \mathrm{a} \tan \theta}{\mathrm{y}-2 \mathrm{a} \tan \theta}+1
$$

Substituting $+40 \mathrm{deg}$ for $\theta$ yields $M=1.147$, and this indicates that a meridional magnifier oriented horizontally over the left eye, with a magnification of $14.7 \%$, would cause a truly horizontal line to appear rotated out of the frontal plane by $40 \mathrm{deg}$, left side away. In effect, a $14.7 \%$ lens over the left eye is the optical equivalent of the +40-deg standard viewed directly without lenses. The original question of the perceptual effect of a $4 \%$ size lens on the +40-deg standard can be resolved into the hypothetical case of the effects of viewing a line target, oriented in the frontal plane, with a $14.7 \%$ lens over the left eye and a $4 \%$ lens over the right eye. For the general case in which lenses are worn over both eyes,

$$
\begin{aligned}
\Delta \eta & =\left(\alpha_{1}-\alpha_{2}\right)-\left(M_{1} \alpha_{1}-M_{2} \alpha_{2}\right) \\
& =\alpha_{2}\left(M_{2}-1\right)+\alpha_{1}\left(1-M_{1}\right) .
\end{aligned}
$$


Table 6

Mean and Standard Errors $\left({ }^{\sigma} \mathrm{m}\right)$ of Absolute and Error Scores for Three Viewing Conditions, i.e., No Lens (NL), Overall Size Lens (OSL), and Inducing Lens (L), and Two Target Orientations (0 Deg and 40 Deg)

\begin{tabular}{|c|c|c|c|c|c|c|c|}
\hline & & \multicolumn{6}{|c|}{ Orientation of Standard } \\
\hline & & \multicolumn{3}{|c|}{$0 \mathrm{Deg}$ (in Frontal Plane) } & \multicolumn{3}{|c|}{40 Deg (Left Side Away) } \\
\hline & & $\begin{array}{l}\text { Mean } \\
\text { Error } \\
\text { Score } \\
\end{array}$ & $\begin{array}{c}\text { Mean } \\
\text { Absolute } \\
\text { Score }\end{array}$ & $\sigma \mathrm{m}$ & $\begin{array}{l}\text { Mean } \\
\text { Error } \\
\text { Score }\end{array}$ & $\begin{array}{l}\text { Mean } \\
\text { Absolute } \\
\text { Score }\end{array}$ & $\sigma \mathrm{m}$ \\
\hline $\begin{array}{l}\text { Viewing } \\
\text { Conditions }\end{array}$ & $\begin{array}{l}\text { NL } \\
\text { OSL } \\
\text { IL }\end{array}$ & $\begin{array}{r}-0.1 \\
-17.7 \\
-0.1\end{array}$ & $\begin{array}{r}-0.1 \\
-17.7 \\
-0.1\end{array}$ & $\begin{array}{r}.63 \\
1.78 \\
.90\end{array}$ & $\begin{array}{l}-1.0 \\
-7.4 \\
-1.0\end{array}$ & $\begin{array}{l}39.0 \\
32.6 \\
39.0\end{array}$ & $\begin{array}{l}1.05 \\
1.60 \\
1.02\end{array}$ \\
\hline
\end{tabular}

Note: - indicates left side toward $S$

Letting $M-1=m$, the percent magnification, then

$$
\Delta \eta=m_{2} \alpha_{2}-m_{1} \alpha_{1}
$$

If $\alpha_{1}=\alpha_{2}$, then $\Delta \eta=\alpha\left(m_{2}-m_{1}\right)$. Since the GE is proportional to $\Delta \eta$, it follows that the GE will be a function simply of the differences in percent magnification between the two lenses. In the present case, this leads to the conclusion that the GE will be equivalent to that produced by a lens of $10.7 \%$ worn over the left eye. For this value, Ogle's formula predicts a frontal plane rotation of about $32 \mathrm{deg}$, left side away. Thus, the GE with the 40-deg target should produce an error of approximately $8 \mathrm{deg}$, left side toward S.

\section{Results}

\section{Screening Task}

Groups $F$ and $T$ showed mean error scores of -.6 and -1.0 (right side away). Analysis of variance showed no significant differences between the groups, nor was the combined mean of -.8 significantly different from zero.

\section{Matching Data, Rightward Displacement}

Means and standard errors for both error scores and absolute settings are represented in the bottom two rows of Table 3. Analysis of variance of absolute scores showed significant effects of orientation, $F(1,22)=$ $450.15, \mathrm{p}<.01$, indicating that $\mathrm{Ss}$ can readily discriminate in their settings, the two target orientations. Displacement was also significant, $F(1,22)=12.73$, $\mathrm{p}<.01$, as well as the interaction of displacement and orientation, $F(1,22)=4.84, p<.05$. The latter two results were identical for the analysis of error scores. However, the error scores did not vary significantly with orientation, $F(1,22)=0.80, p>.05$. These results indicate that displacement was effective, but primarily with the standard oriented at $40 \mathrm{deg}$, left side away. Since the comparison was set at $6.8 \mathrm{deg}$ left side toward $S$ relative to its correct position, it can be concluded that the standard appeared to be underrotated by that amount. Such an error is consistent with an underestimation in apparent angular displacement and with the results of the previous experiment. The results with the frontal plane standard are in the same direction, but considerably reduced over that obtained in Experiment II. This is attributable probably to random sampling, ${ }^{11}$ the low negative mean being due mainly to two large positive entries out of a total $\mathrm{N}$ of 12 in that condition.

\section{Pointing Data}

Results of the pointing task are represented in the bottom two rows of Table 4 . Analysis of variance of the deviations from a pointing position parallel with the median plane, i.e., the absolute scores, showed significant effects of target displacement, $F(1,22)=$ $395.99, \mathrm{p}<.01$, but no significant effect of orientation, $F(1,22)=0.01, p>.05$, or of the interaction, $F(1,22)=$ $0.54, p>.05$. This outcome is consistent with that of Experiment II in indicating that Ss readily discriminated the displacement positions in their pointing responses. Analysis of the pointing errors, i.e., deviation from the correct pointing direction, show identical $\mathrm{F}$ ratios as the absolute scores for orientation and the interaction term. However, the errors did not vary significantly with displacement, $F(1,22)=0.39, \mathrm{p}>.05$. Thus, as in the previous experiment, a constant error to the right is in evidence.

In order to examine the pointing responses for evidence of underestimation of apparent displacement, the angular differences in the absolute pointing direction between the straight-ahead and (rightward) displaced targets was determined. The obtained values, represented in Table 5, were 28.9 and $31.0 \mathrm{deg}$ for the frontal and rotated targets, respectively. The actual angular shift was $29 \mathrm{deg}$ in each instance. As in the case of Experiment II, the obtained values are remarkably close to the calculated ones, and hence the conclusion is supported that except for the constant error, the pointing measure is a remarkably accurate indicator of relative angular displacement.

\section{Matching with Aniseikonic Lenses}

Matching data for the three observation conditions are represented in Table 6 . Although Condition IL was run 
after the NL and OSL matches were made, the three conditions were analyzed together. Analysis of variance of the absolute scores showed significant effects of orientation. $F(1,22)=1,492.68$, viewing conditions, $F(2,44)=70.26$, and the interaction of the two, $F(2,44)$ $=15.38$. In all cases, $p<.01$. The analysis of errors yielded identical results for the latter two variables given above. In addition, errors varied significantly as a function of target orientation, $F(1,22)=6.47, p<.05$. These analyses indicate, first, that for any given orientation, NL and IL were strictly equivalent, i.e., there was no evidence whatsoever for an induced effect. Second, the analyses show that performance under NL and IL was veridical, i.e., Ss were accurate in the matching task. By contrast, the nonveridical matches produced by the introduction of the OSL effects were close to those predicted on the basis of the analysis of binocular disparity, i.e., the OSL enrors for the two orientations differed significantly. Thus, for the frontal standard, a rotation of -17.7 (right side away) was obtained, whereas $-14 \mathrm{deg}$ was predicted. A value of $32 \mathrm{deg}$ (left side away) was predicted for the rotated target, and $32.6 \mathrm{deg}$ was obtained. In neither case, was there a significant difference between the obtained and predicted values. Clearly, no evidence for an induced effect under OSL viewing was present.

\section{DISCUSSION}

In Experiments $I$ and II, displacement of the target in the median plane produced near perfect matches, i.e., there were no significant effects on apparent orientation. In the case of these vertically oriented targets, it is possible to account for a veridical match on the basis of cyclofusional eye movements. Since targets rotated top away represent stimuli for excyclotorsion, while those oriented top toward $\mathbf{S}$ induce incyclotorsion (Harker, 1966), then, assuming the optical equivalence of displaced and rotated targets, a vertical line displaced above eye level should represent a stimulus for excyclotorsion, one displaced below should induce incyclotorsion. These changes in the torsional positions of the eyes would cause the displaced targets to stimulate the retina at corresponding points, in the same fashion as a nondisplaced vertical target viewed at eye level. Thus, both displaced and nondisplaced targets would produce equivalent retinal patterns and both would be perceived as upright simply on the assumption that images parallel to the vertical retinal meridians will lead to the perception of a vertical target. Based upon a similar line of reasoning, Ogle (1964) suggested, "In order that vertical objects such as walls, trees and so forth may maintain their orientation as one raises or lowers the eyes to look at various parts, a cyclofusional movement is a necessity [p. 118]." Although such a mechanism could account for the near veridical matches of the vertically oriented target, the equally precise settings of the 40-deg (top away) target cannot be explained in the same manner. If cyclofusional movements did occur with this target, they would have served the function of reducing the binocular disparity, thereby causing the line to appear less tilted. However, reference to Table 2 shows no evidence for such an effect. The interpretation of the obtained orientation constancy in terms of a compensation for the direction of gaze is thereby sustained, although the role of torsional eye movements in the present context is not entirely clear. It is conceivable that they are, in fact, quite negligible (Kertesz, 1972; Kertesz \& Jones, 1970).

The induced effect, as a possible alternative interpretation to compensation with horizontal stimuli, already has been discussed, with the conclusion that the present line stimuli simply were not suitable to produce the IE. Thus, in the case of the horizontally displaced targets also, compensation remains as a likely explanation. A shortcoming to this approach was the failure of the pointing data with horizontal stimuli to support the interpretation of the matching errors in terms of an underestimation in the apparent displacement. Indeed, in general, pointing errors did not correlate significantly with matching errors. This is, of course, a familiar outcome in studies of the perceptual constancies where estimates of phenomena, and of their conditional events frequently fail to correlate, as, e.g., size and distance estimates (Gruber, 1954), or apparent object orientation with estimates of body orientation (Ebenholtz, 1972), etc. The solution would seem to lie in the possibility that the conditional event (e.g., apparent displacement) is dependent upon factors not all of which exert an equal influence on the phenomenon itself (e.g., apparent orientation). This view is supported by the fact that pointing errors tended to be influenced by target orientation (Experiment III), whereas matching errors were free of such effects. Thus, the use of pointing as a measure of apparent displacement may itself have contributed to the poor correlation between apparent displacement and orientation.

The tendency to point below the vertical targets or to the right of horizontal targets has prevented the direct interpretation of pointing errors in terms of errors in apparent displacement. Perhaps a more symmetrically located wrist position, or counterbalancing over left and right limbs, would eliminate or reduce the constant errors obtained with the present procedure. Despite these shortcomings, it is worth noting that the angular difference in pointer settings corresponding to the difference between straight ahead and displaced target positions, was with minor exception remarkably accurate. As Table 5 indicates, the wrist-rotation angle required to point correctly at the peripheral targets, i.e., the calculated angle, was within $3 \mathrm{deg}$ of the obtained settings in 7 out of 10 instances shown. This is evidence for a sensory-motor constancy process in its own right, quite apart from the constancy of object orientation. It would appear that the pointing at visually fixated targets entails first of all a readout of the ocular rotation angle, 
perhaps as a difference from the primary position. This is the converse of the findings of Steinbach (1969) and Steinbach and Held (1968), who have shown that efferent signals to the arm convey spatial information that is available to the oculomotor system. In addition to monitoring ocular position, however, information regarding the elbow angle and the angle of the upper arm relative to the trunk, etc., must be used to condition the final choice of wrist angle. ${ }^{12}$ In the present study, although a constant error was present, the relative angular position of the targets was indeed quite faithfully recorded in the pointing record. This outcome suggests that the pointing procedure does provide an indicator of relative egocentric direction, but that constant errors may emerge because of errors in the registered position of parts of the limb. For example, pointing too far to the right of horizontal targets could be caused by the improper proprioception that the arm is too far to the left of the median plane. Similarly, pointing below vertical targets could result from the mistaken sense that the arm is too high. Thus, while the pointing procedure, in its present form, was not wholly adequate to measure apparent displacement, it has proved to be an interesting source of speculation about sensory-motor constancy.

In the present experiments, ocular fixation was neither monitored nor controlled. Therefore, the role of eye movements as potential sources of information about target displacement and orientation remains to be determined.

The results of the present experiments would seem to be relevant to two research domains of space perception, viz, the psychophysics of binocular vision and the study of the perceptual constancies. The major implication to be drawn is that $S$ does not, and perhaps cannot, simply match binocular disparities, but rather that $S$ utilizes visual inputs and their conditional states according to some algorithm. Thus, in the present instance, binocular disparity and apparent displacement are evaluated according to an algorithm that permits the deduction of apparent orientation. This outcome, in turn, can be set to match some predetermined criterion such as frontoparallelarity or some other specified orientation. The joint processing of retinal disparity and displacement angle is presumed to underlie orientation constancy, as exhibited under the circumstances of the present investigation. It is likely that the same interpretation holds for the horopter studies of the apparent frontoparallel plane in asymmetrical convergence. ${ }^{13}$

\section{REFERENCES}

Day, R. H., \& Wade, N. J. Mechanisms involved in visual orientation constancy. Psychological Bulletin, 1969, 71, 33-42.

Ebenholtz, S. M. Perception of the vertical with body tilt in the median plane. Journal of Experimental Psychology, 1970, 83, $1-6$.
Ebenholtz, S. M. The constancy of object orientation: Effects of target inclination. Psychologische Forschung, 1972, 35, 178-186.

Gillam, B. J. Perception of slant when perspective and stereopsis conflict: Experiments with aniseikonic lenses. Journal of Experimental Psychology, 1968, 78, 299-305.

Gruber, H. E. The relation of perceived size to perceived distance. American Journal of Psychology, 1954, 67, 411-426.

Harker, G. S. Retinal correspondence and the perceived vertical. Perceptual \& Motor Skills, 1966, 23, 347-360.

Hill, A. L. Direction constancy. Perception \& Psychophysics, $1972,11,175-178$.

Kertesz, A. E. The effect of stimulus complexity on human cyclofusional response. Vision Research, 1972, 12, 699-704.

Kertesz, A. E., \& Jones, R. W. Human cyclofusional response. Vision Research, 1970, 10, 891-896.

Ogle, K. N. Researches in binocular vision. New York: Hafner, 1964.

Shipley, T., \& Rawlings, S. C. The nonius horopter-I. History and theory. Vision Research, 1971, 10, 1225-1262.

Steinbach, M. J. Eye tracking of self-moved targets: The role of efference. Journal of Experimental Psychology, 1969, 82, 366-376.

Steinbach, M. J., \& Held, R. Eye tracking of observer-generated target movements. Science, 1968, 161, 187-188.

Wallach, H., \& Zuckerman, C. The constancy of stereoscopic depth. American Journal of Psychology, 1963, 76, 404-412.

\section{NOTES}

1. Since the convergence distance to the displaced stimulus is greater than to the comparison, equally spaced points along the stimulus surface project smaller disparities in the former case than in the latter. Consequently, disparity matching actually might require the comparison to be rotated somewhat less than the standard.

2. Distance to the target would also be a factor in the interpretation of the disparities (Wallach \& Zuckerman, 1963), and hence a contributor to orientation constancy.

3. The actual shape of the nonius horopter would be critical where $S$ matches the orientation of a displaced target seen in peripheral vision while fixating with the eyes in symmetric convergence.

4. This is also referred to as the problem of the stability of corresponding points, i.e., the question of whether or not $S$ uses the identical retinal elements, in adjusting a horopter apparatus according to some criterion such as the apparent frontoparallel plane, under symmetrical and asymmetrical convergence.

5 . One $\mathrm{S}$ with a mean error of $6.5 \mathrm{deg}$ was inadvertently left in the sample.

6. This results from the fact that in the pointing task, for any given displacement angle, the difference between the means of the two orientation conditions is the same whether measured in terms of error scores or in absolute scores. In the matching task, however, for any given orientation condition, the differences between the three displacement means is the same for both methods of measurement.

7. Matching errors made with the standard at the central position were subtracted from the errors produced at the displaced positions. The net result was a relative error analogous to the procedure used to determine the relative error in pointing.

8 . This is a plane normal to the bisector of the interocular axis at the point of fixation.

9. Actually, there was a magnification of $6.1 \%$ based upon the relative distances between the left and right eyes, respectively, and the fixation point on the displaced target. Nevertheless, the $4 \%$ lenses used were quite sufficient for exhibiting an IE. 
10. The assumption was made that the rotation is influenced solely by the horizontal component of the overall magnification. However. it probably is the case that vertical and even oblique components may contribute by degrading the effectiveness of the transverse disparities.

11. Ss were not screened for aniseikonia, yet this condition could contribute significantly to the matches.

12. For directions in a horizontal plane, this angle is determined by two distances. One of which is the lateral displacement of the wrist to the right or left of S's median plane. The second is the distance in depth between S's frontal plane and the target. Both of these distances are encoded in terms of the various limb and joint positions.

13. Because of this tendency toward constancy, we agree with the view of Shipley \& Rawlings (1971) that the true horopter must be based upon the criterion of common visual direction, i.e., the nonius horopter, and that this is the "... only reliable and direct path to the distribution of corresponding retinal points, as manifest subjectively ... [p. 1241]."

(Received for publication August 21, 1972; revision received June 19,1973 .) 